\title{
Determination of trapping parameters in poly(p-phenylenevinylene) light-emitting devices using thermally stimulated currents
}

Cite as: Journal of Applied Physics 84, 87 (1998); https://doi.org/10.1063/1.368004

Submitted: 13 October 1997. Accepted: 25 March 1998. Published Online: 19 June 1998

M. Meier, S. Karg, K. Zuleeg, W. Brütting, and M. Schwoerer

ARTICLES YOU MAY BE INTERESTED IN

Space-Charge-Limited Currents in Organic Crystals

Journal of Applied Physics 33, 205 (1962); https://doi.org/10.1063/1.1728487

Electron and hole transport in poly(p-phenylene vinylene) devices

Applied Physics Letters 68, 3308 (1996); https://doi.org/10.1063/1.116583

Deep-level transient spectroscopy: A new method to characterize traps in semiconductors Journal of Applied Physics 45, 3023 (1974); https://doi.org/10.1063/1.1663719
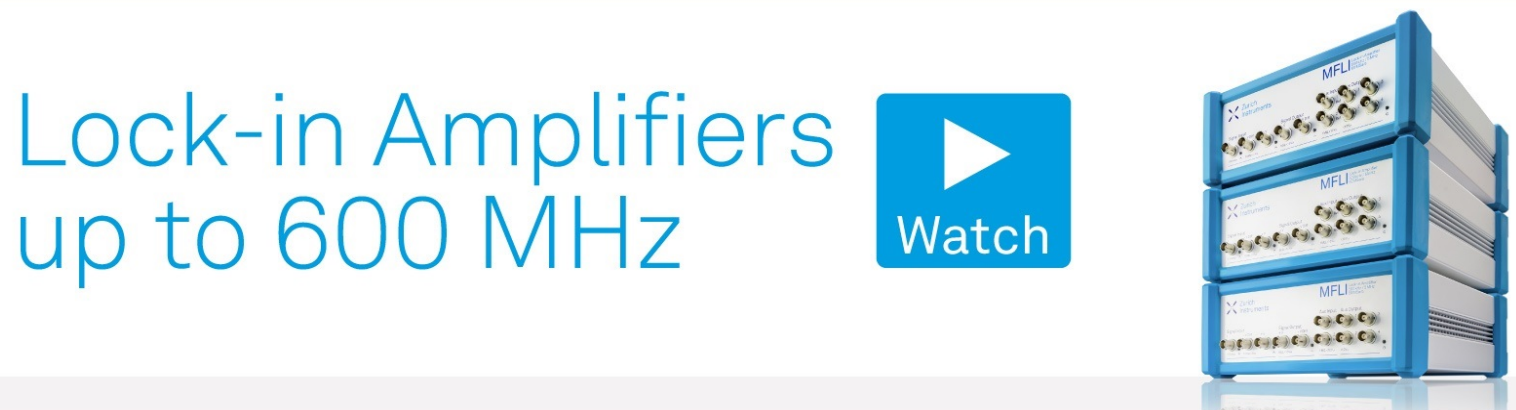


\title{
Determination of trapping parameters in poly(p-phenylenevinylene) light-emitting devices using thermally stimulated currents
}

\author{
M. Meier, S. Karg, ${ }^{\text {a) }}$ K. Zuleeg, W. Brütting, ${ }^{\text {b) }}$ and M. Schwoerer \\ Experimental Physics II, University of Bayreuth, 95440 Bayreuth, Germany
}

(Received 13 October 1997; accepted for publication 25 March 1998)

\begin{abstract}
Employing thermally stimulated current (TSC) technique, the existence of distinct trap distributions in poly(p-phenylenevinylene) (PPV) light-emitting devices has been established. In devices with an indium tin-oxide (ITO) anode two TSC peaks in the temperature range between 100 and $150 \mathrm{~K}$ are observed. They correspond to trap levels with a depth of $0.03-0.06 \mathrm{eV}$ and $0.13-0.18 \mathrm{eV}$, respectively. The total density of these trap species is of the order of $10^{16} \mathrm{~cm}^{-3}$ which is in good agreement with the dopant concentration obtained from capacitance-voltage measurements. The investigated peaks in the TSC spectrum do not occur if a Au electrode is used instead of ITO. Hence, the reaction of ITO with the elimination products (mainly $\mathrm{HCl}$ ) during the conversion of the PPV precursor leads to the formation of these shallow traps. Deeper trap states with energies between 0.6 and $1 \mathrm{eV}$ have been detected, too. The latter trapping levels appear independently of the anode substrate material and are due to the influence of air. (c) 1998 American Institute of Physics. [S0021-8979(98)02313-5]
\end{abstract}

\section{INTRODUCTION}

The electronic conductivity of poly(p-phenylenevinylene) (PPV) prepared by the precursor route changes by numerous orders of magnitude when doped with electron acceptors. ${ }^{1,2}$ Already the exposure to air increases the conductivity $\sigma$ of PPV (converted at $300{ }^{\circ} \mathrm{C}$ in vacuum) within seconds from less than $10^{-15} \mathrm{~S} / \mathrm{cm}$ to about $10^{-13} \mathrm{~S} / \mathrm{cm}$ at room temperature. ${ }^{3}$ The activation energy $E_{a}$ obtained from the temperature dependence of $\sigma(T)$ also changes from 0.9 $\mathrm{eV}$ of pristine PPV to $0.5 \mathrm{eV}$ of PPV exposed to air. ${ }^{3,4}$ The adsorbed oxygen can be removed by annealing under vacuum indicating a reversible doping process.

PPV prepared by the precursor route on an indium-tinoxide (ITO) substrate, a technique often used for the preparation of polymer light-emitting devices (LEDs), is subject to additional chemical reactions of the precursor PPV leaving groups with the ITO substrate. Especially, the reaction of $\mathrm{HCl}$ with indium (or indium oxide) leads to the formation of the oxidizing agent $\mathrm{InCl}_{3} .{ }^{5}$ This reaction leads to doping of the PPV film, which has been proven and investigated by current-voltage characteristics and capacitance-voltage measurement on LEDs $^{6-8}$ as well as with analytical methods such as secondary ion mass spectroscopy, scanning electron microscopy, and energy dispersive $\mathrm{x}$-ray analysis. ${ }^{5,9}$ The $\mathrm{p}$ type doping causes in Al/PPV/ITO LEDs the formation of a Schottky barrier at the Al/PPV interface and an ohmic contact with low impedance at the PPV/ITO interface.

The subject of this article is to gain more information about the dopant and trap species, especially their energy levels, by means of thermally stimulated currents (TSC). After carefully testing whether this method can be used for the

\footnotetext{
${ }^{a)}$ Present address: Electronic Materials, Darmstadt University of Technology, Petersenstr. 23, 64287 Darmstadt, Germany.

${ }^{b}$ Electronic mail: wolfgang.bruetting@uni-bayreuth.de
}

characterization of PPV LEDs, we investigate the influence of different anode substrates and conversion conditions of the PPV precursor as well as of the environmental conditions on the trap states.

\section{EXPERIMENTAL DETAILS}

Thermally stimulated current measurements were performed in a continuous flow cryostat (Oxford Instruments CF1200) using liquid helium or nitrogen. Two samples could be measured simultaneously using two independent electrometers (Keithley 617) for better comparison of different device configurations and fabrication procedures. The temperature was measured with an $\mathrm{Au}-\mathrm{Fe} / \mathrm{Chromel}$ thermocouple placed close to the samples and controlled with a temperature controller. The temperature range of the setup is 5 to $350 \mathrm{~K}$ and constant heating rates up to $10 \mathrm{~K} / \mathrm{min}$ are achievable. After mounting the samples into the cryostat, the sample chamber was evacuated to $10^{-5} \mathrm{mbar}$ and filled with $\mathrm{He}$ contact gas. The cryostat was cooled down to $5 \mathrm{~K}$ (or 80 $\mathrm{K}$ in the case of liquid nitrogen as coolant). At this temperature, traps were filled by applying a constant current or voltage to the sample for a certain time (typically $5 \mathrm{~min}$ ). After the trap filling procedure, the TSC measurements were performed in short circuit mode in a two-probe configuration. The measurement limit of the setup was less than $10^{-13} \mathrm{~A}$. The samples were heated with a constant heating rate $\beta$ $=d T / d t$ between 3 and $10 \mathrm{~K} / \mathrm{min}$. In preliminary measurements we ensured that the observed peaks in the TSC spectra of samples with PPV originate from thermally induced detrapping of carriers. If the peak position at a given heating rate reflects the depth of a certain trap species it will not depend on external parameters like filling conditions, starting temperature $T_{0}$ or sample thickness. We carefully checked the spectra under several experimental conditions and could demonstrate the demanded independence. 
PPV was synthesized via the precursor route as described by Herold et al. ${ }^{10}$ Films were cast with a draw bar technique onto the anode substrates which consisted either of commercial indium-tin-oxide substrates or of glass substrates coated with Au. The PPV precursor was converted onto the anode substrates at about $165^{\circ} \mathrm{C}$ in vacuum $\left(10^{-2}\right.$ mbar $)$ or under argon atmosphere. On top of the devices, an Al cathode was evaporated at $10^{-6}$ mbar. While the samples were mounted into the cryostat, they were exposed to air for less than $1 \mathrm{~h}$. All devices investigated here had an active area of $0.25 \mathrm{~cm}^{2}$.

\section{ANALYSIS OF TSC SPECTRA}

The method of TSC has been widely used to determine the density and energy distribution of trap states in inorganic semiconductors, organic molecular crystals, and polymeric photoconductors. ${ }^{11-14}$ The experiment consists of filling the trap states at low temperature either by passing a current through the sample or by photoexcitation, then heating the sample in the dark at a constant rate with or without external field. As the traps are released, a current is measured and characteristic peaks are observed. By integrating the released current over time, the amount of trapped charges is obtained and knowing the volume of the sample one can estimate the average trap density.

The origin of the detected peaks depends on the transport properties of the investigated material. In the conventional semiconductor model, the TSC peaks arise from the increased number of free carriers which are released thermally by detrapping. So, the temperature where a current peak occurs is correlated to the energetic depth of the corresponding trap state. In hopping transport systems, carriers move by thermally assisted tunneling from one localized state to another without ever moving in extended band states. According to a model proposed by Chen, ${ }^{15}$ the detected TSC peak is interpreted as thermally induced time-of-flight of carriers. This approach allows one to derive information on the thermally activated mobility of charge carriers in the solid. As a consequence of this interpretation the peak position on the temperature scale is field and thickness dependent. Furthermore, hopping of one type of carriers should give rise to only a single-peak TSC curve, except for charge carrier groups with significantly different mobilities. This theory has been successfully applied to polyvinylcarbazole as model system for hopping transport. ${ }^{11}$

The transport process of PPV should be polaronic. ${ }^{16}$ The measured charge carrier mobilities are relatively low $\left(10^{-5} \mathrm{~cm}^{2} / V s \text { at } F=10^{5} \mathrm{~V} / \mathrm{cm} \text { and } 300 \mathrm{~K}\right)^{17}$ and thus in a range typical for materials with predominating hopping transport. However, for our data analysis we followed the classical approach from Haering and Adams, ${ }^{18}$ and Cowell and Woods, ${ }^{19}$ since our measurements indicate no significant thickness dependence of the TSC peak position. Furthermore, we detected several peaks which are partially enhanced while storing the sample in air. These are strong hints that the measured peaks arise from detrapping of carriers from distinct trap levels and not from thermally induced time-of-flight.
The essential idea of the TSC technique can be understood by considering the simplest case of unipolar carrier trapping in one discrete trap level. ${ }^{18,19}$ The following description is valid for electron traps but can be transferred to hole traps, too. The probability $\tau_{t}^{-1}$ for detrapping an electron from a trapping state is related to the trap depth $\Delta E$ $=E_{C}-E_{t}$ by

$$
\tau_{t}^{-1}=\nu \exp [-(\Delta E) / k T],
$$

where $\nu$ is the "attempt-to-escape" frequency, $E_{C}$ the conduction band edge and $E_{t}$ the trap energy. After the charging process the densities of free carriers $n(t)$ and trapped carriers $n_{t}(t)$ are given by

$$
\frac{d n}{d t}=-\frac{d n_{t}}{d t}-\frac{n}{\tau}
$$

if a spatial variation of $n_{t}$ is neglected. The first term on the right hand side represents the rate of change of the trapped electron density, and the second term the recombination of free charge carriers where $\tau$ is the average lifetime of a free carrier until recombination. The variation of the trapped carrier density $d n_{t} / d t$ can be expressed as

$$
\frac{d n_{t}}{d t}=-\frac{n_{t}}{\tau_{t}}+n\left(N_{t}-n_{t}\right) S_{t} v,
$$

where $v$ is the thermal velocity, $S_{t}$ the trap capture cross section and $N_{t}$ the total concentration of available traps. If one assumes that the free electron lifetime $\tau$ is small as compared to the residence time of the trapped charge carrier, which means that detrapped electrons recombine almost immediately and retrapping is negligible, Eqs. (2) and (3) reduce in the so-called case of slow retrapping to

$$
\frac{n}{\tau}=-\frac{d n_{t}}{d t}=\frac{n_{t}}{\tau_{t}}
$$

Finally, the thermally stimulated conductivity is obtained as

$$
\sigma=e \mu n=-e \mu \tau \frac{d n_{t}}{d t}
$$

where $e$ is the elementary charge and a constant mobility $\mu$ is assumed. If the specimen is heated at a constant rate $\beta=d T / d t$, integration of Eq. (4) gives $n_{t}(T)$ and finally,

$$
\sigma(T)=n_{t_{0}} \tau e \mu \nu \exp \left[-\frac{\Delta E}{k T}-\int_{T_{0}}^{T} \frac{\nu}{\beta} \exp \left(-\frac{\Delta E}{k T^{\prime}}\right) d T^{\prime}\right],
$$

where $n_{t_{0}}$ denotes the density of filled traps at the initial temperature $T_{0}$. Cowell and Woods have shown that repeated integration by parts of the integral in Eq. (6) leads to a convergent infinite series. Since $\Delta E / k T>1$ is valid in all practical cases, a good approximation for $\sigma(T)$ can be obtained then by skipping all but the first term in the series. Equation (6) then becomes: ${ }^{19}$

$$
\sigma=A \exp \left[-\Theta-B \exp (-\Theta) \Theta^{-2}\right]
$$

with $A=n_{t} \tau e \mu \nu, B=\nu \Delta E / \beta k$ and $\Theta=\Delta E / k T$. $B$ is obtained through differentiation of Eq. (7): 


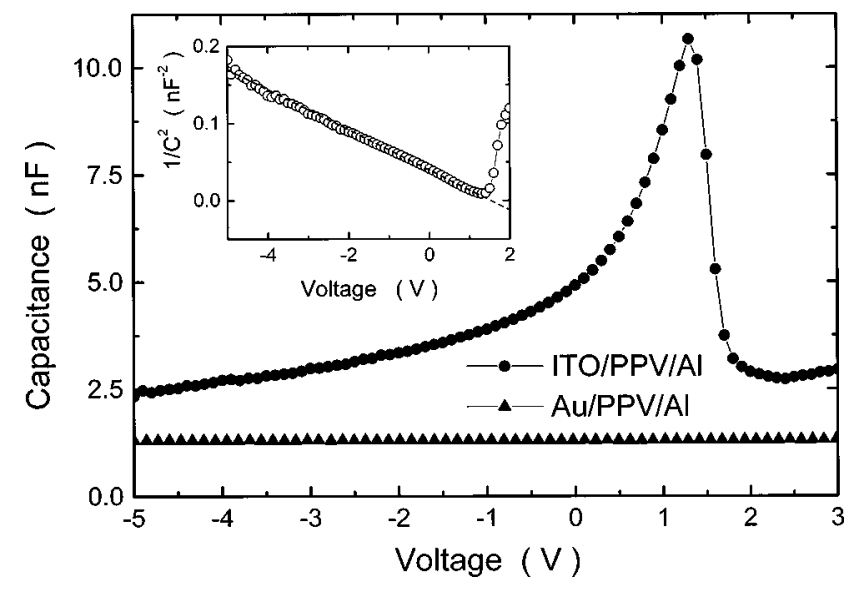

FIG. 1. Comparison of capacitance-voltage characteristics of an ITO/ PPV/Al and a Au/PPV/Al device. The inset shows the $1 / C^{2}$ plot for the ITO/PPV/Al device together with a linear fit yielding the ionized acceptor density. Measurements were performed with a Hewlett Packard 4194A impedance analyzer at a test frequency of $111 \mathrm{~Hz}$ (for details see Ref. 7).

$$
B=\exp \left(\Theta_{m}\right) \Theta_{m}^{2},
$$

where $T_{m}$ represents the temperature at the peak maximum and $\Theta_{m}=\Delta E / k T_{m}$. Equations (7) and (8) were used for numerical curve fitting with three fitting parameters $T_{m}, A$ and $\Delta E$. In our evaluation we concentrate only on the energetic depth of the observed trap states, since we do not have sufficient information on the capture cross section and carrier lifetimes in PPV.

In the above model, the rate equation (3) was solved in the limit of slow retrapping $\left[\tau^{-1} \gg\left(N_{t}-n_{t}\right) S_{t} v\right]$ which is appropriate for fast electron-hole recombination. In the limit of fast retrapping $\left[\tau^{-1} \ll\left(N_{t}-n_{t}\right) S_{t} v\right]$, the solution of the rate equation leads to expressions for the thermally stimulated conductivity which are very similar to Eqs. (6) and (7). ${ }^{19}$ Only the peak width is slightly influenced but not the position of the peak. As expected, a test of fitting the measured data with equations for both retrapping kinetics gave no significant differences for the obtained parameters.

\section{EXPERIMENTAL RESULTS AND DISCUSSION}

\section{A. Doping of PPV by the ITO substrate}

Our investigations on light-emitting diodes based on precursor PPV applying electrical methods as well as analytical techniques have shown that chemical reactions of the precursor leaving groups (especially $\mathrm{HCl}$ ) with the underlying substrate can occur during the thermal conversion. ${ }^{5-9}$ Depending on the reactivity of the substrate material, device characteristics can vary between a Schottky diode and a metalinsulator-metal device. This indicates that the density of ionizable states within the band gap depends on the device preparation conditions. The most direct evidence for doping of PPV during the thermal conversion process comes from capacitance-voltage measurements in reverse-bias direction, which are shown in Fig. 1 for ITO and Au substrates. The fabrication on $\mathrm{Au}$ substrates leads to insulating material with a voltage independent capacitance corresponding to the dielectric constant of the polymer. In contrast, PPV devices

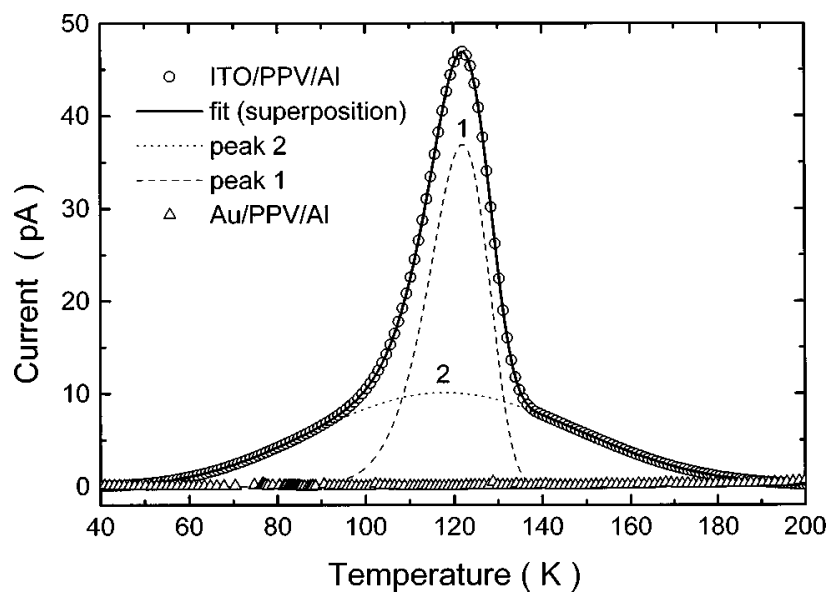

FIG. 2. Comparison of TSC spectra of an ITO/PPV/Al and a Au/PPV/Al device. Both samples were measured simultaneously after identical trap filling. The spectrum of the ITO device can be fitted after Cowell and Woods (see Ref. 19) by a superposition of two peaks.

fabricated on ITO substrates show the behavior of a doped organic semiconductor with a strongly voltage dependent capacitance $C(V)$. The linear dependency of $1 / C^{2}$ vs voltage (for $V<1.3 \mathrm{~V}$ ) shown in the inset of Fig. 1 suggests that the device capacitance can be described in analogy to conventional semiconductor Schottky junctions: ${ }^{20}$

$$
C=A \sqrt{\frac{e \epsilon_{0} \epsilon N_{A}}{2\left(V_{D}-V\right)}},
$$

where $A$ is the device area, $\epsilon_{0}$ the vacuum permittivity, $\epsilon$ the high frequency dielectric constant, $N_{A}$ the ionized acceptor concentration and $V_{D}$ the diffusion voltage. Using this equation the data shown in Fig. 1 yield an ionized acceptor density of $N_{A}=2.3 \times 10^{16} \mathrm{~cm}^{-3}$. Evaluation of a series of samples from different polymer batches gives dopant concentrations typically in the range of $10^{16}-10^{17} \mathrm{~cm}^{-3} \cdot{ }^{7,8} \mathrm{Re}-$ cent temperature dependent studies of impedance spectra have furthermore revealed that the temperature dependence of the acceptor density can be described with an acceptor energy between 0.16 and $0.2 \mathrm{eV}$ relative to the valence band. $^{21}$

In order to get independent information about the density and energy levels of states created by the chemical reaction of the PPV precursor leaving groups with the substrate, we performed TSC measurements on devices with different anodes. Figure 2 shows a comparison of TSC spectra of ITO/ $\mathrm{PPV} / \mathrm{Al}$ and $\mathrm{Au} / \mathrm{PPV} / \mathrm{Al}$ devices in the temperature range from 40 to $200 \mathrm{~K}$. Both samples had a PPV layer thickness of about $600 \mathrm{~nm}$ and the trap filling was performed identically, passing a current of $10 \mu \mathrm{A}$ at $10 \mathrm{~K}$ for $5 \mathrm{~min}$ through the device. The curves were recorded at a heating rate of about $5 \mathrm{~K} / \mathrm{min}$. While there is no TSC signal detectable within our experimental resolution $\left(10^{-13} \mathrm{~A}\right)$ when $\mathrm{Au}$ is used as anode, the sample with ITO clearly shows a peak at about $122 \mathrm{~K}$. Thus, the elimination of PPV on ITO substrates creates at least one trap state in the polymer which can be detected with TSC. The TSC spectrum of the ITO sample was analyzed by the method of Cowell and Woods. A suitable fit can be obtained by a superposition of two TSC peaks 


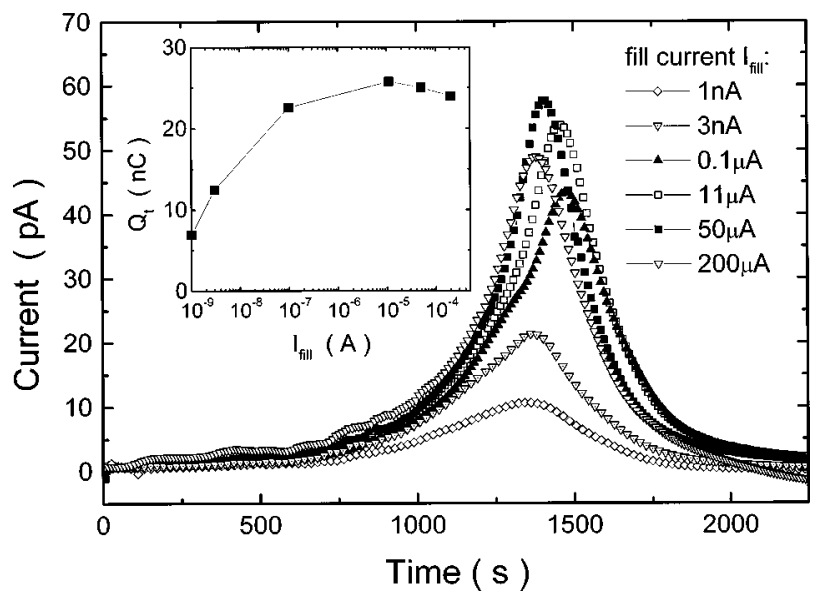

FIG. 3. TSC spectra of an ITO/PPV/Al device for different trap filling currents. The inset shows the dependence of the released amount of charges on the fill current.

as shown by the solid line in Fig. 2. The least-squares fit gives a trap depth of $\Delta E=0.18 \mathrm{eV}\left(T_{\max }=122 \mathrm{~K}\right)$ for the narrow peak 1 . For the second underlying broader peak 2, a trap depth of about $\Delta E=0.03 \mathrm{eV}$ is obtained. The values vary from sample to sample typically between 0.03 and 0.05 $\mathrm{eV}$ for peak 2 and for peak 1 between 0.13 and $0.18 \mathrm{eV}$.

The integral of the TSC current $I(t)$ over time $t$ yields the amount of detrapped charges $Q_{t}$. If all traps are filled at low temperature, the integral gives the density of releasable traps. Hence, the peak height of the thermally stimulated current depends on the degree of trap filling as well, as it is shown in Fig. 3. All spectra therein were obtained from a single ITO/PPV/Al sample where trap filling was performed with different currents $I_{\text {fill }}$. The fill current was applied for 5 min and the heating rate was $5 \mathrm{~K} / \mathrm{min}$ in all cases. The peak height $I_{\max }$ increases with increasing fill current from about $10 \mathrm{pA}$ at $I_{\text {fill }}=1 \mathrm{nA}$ to $58 \mathrm{pA}$ at $I_{\text {fill }}=50 \mu \mathrm{A}$. At a fill current of $I_{\text {fill }}=200 \mu \mathrm{A} I_{\max }$ dropped to $45 \mathrm{pA}$. In the insert of Fig. 3 the released charge $Q_{t}=\int I(t) d t$ is drawn as a function of the fill current $I_{\text {fill }}$ on a logarithmic scale. $Q_{t}$ saturates at a level of $25 \times 10^{-9} \mathrm{C}$ which corresponds to an average density of releasable traps of $n_{t}=1.2 \times 10^{16} \mathrm{~cm}^{-3}$. This is about the same density as the dopant concentration $N_{A}$ which we have obtained from the capacitance-voltage measurements shown in Fig. 1 and reported previously. ${ }^{7,8}$ These numbers suggest that the ITO related trap states are responsible for the $p$-type doping of PPV and hence for the observed Schottkydiode behavior of ITO/PPV/metal LEDs. In the case of the Au devices, no such peak structure is observable in this temperature range, which correlates well with the absence of a voltage dependent capacitance and an increased bulk resistance of the LEDs with Au anode. The dopant concentration in PPV converted on $\mathrm{Au}$ is estimated to be below $10^{15} \mathrm{~cm}^{-3} \cdot 8$

\section{B. Influence of the conversion conditions}

The method of thermally stimulated currents is even sensitive enough to detect differences in the elimination conditions of PPV. Figure 4 depicts the TSC curves of two PPV

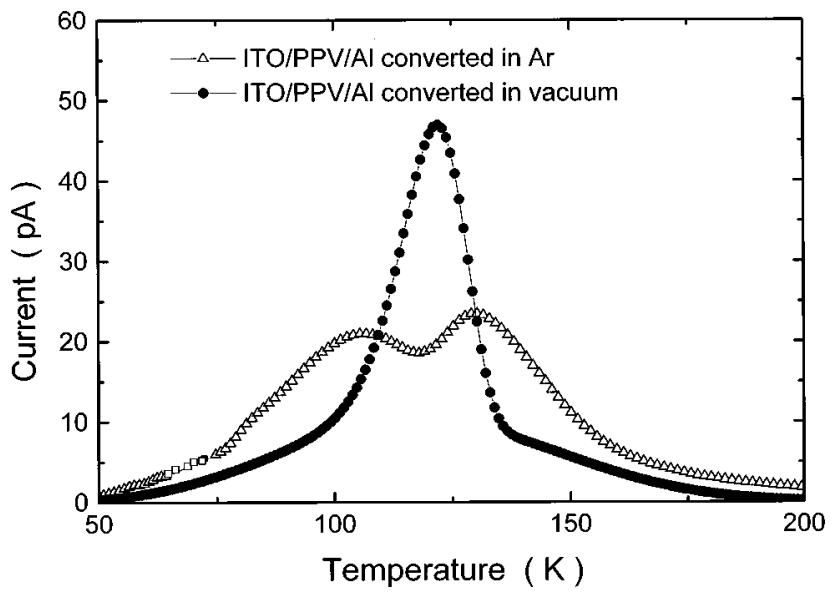

FIG. 4. TSC spectra of ITO/PPV/Al devices for different conversion conditions. Both samples were measured simultaneously after identical trap filling.

LEDs in the configuration ITO/PPV/Al which differ from each other only by the conversion conditions of the PPV precursor. One sample (circles) was converted under vacuum $\left(10^{-2} \mathrm{mbar}\right)$ and the second one (triangles) in argon atmosphere, both at $165^{\circ} \mathrm{C}$ for $2 \mathrm{~h}$. The TSC curve of the sample eliminated under vacuum is already described in Fig. 2. In contrast, the TSC spectrum of the device with PPV converted in Ar atmosphere reveals two clearly separated peaks at about 105 and $130 \mathrm{~K}$. The curve fit leads to corresponding trap energies of $\Delta E=0.06 \mathrm{eV}\left(T_{\max }=105 \mathrm{~K}\right)$ and $\Delta E$ $=0.13 \mathrm{eV}\left(T_{\max }=130 \mathrm{~K}\right)$. The fact that these peaks are broadened as compared to the TSC spectrum of the vacuum eliminated sample is a hint for a broader energetic distribution of trap states in the material converted in argon. The amount of released charge carriers and therefore the estimated trap densities are nearly the same in both devices. Thus it is not astonishing that the PPV devices fabricated under different conversion conditions do not show significant differences in their electrical behavior (current-voltage and capacitance-voltage measurements). ${ }^{6,7}$

\section{Environmentally induced traps}

In the TSC spectra shown so far in this article data were only plotted up to a temperature of $200 \mathrm{~K}$. Between 200 and $300 \mathrm{~K}$ further peaks appear in the spectra independent of the chosen anode substrates. As an example, we show in Fig. 5 the complete TSC spectrum of an ITO/PPV/Al device (PPV converted in Ar atmosphere) between 5 and $250 \mathrm{~K}$. In addition to the already discussed peaks originating from the reaction with the ITO substrate (peaks 1 and 2), there are two narrow peaks in the temperature range above $200 \mathrm{~K}$. The corresponding trap energies are about 0.9 and $1.0 \mathrm{eV}$ for peaks 3 and 4, respectively. We have found that the relative peak height, width and position can vary from sample to sample and also depend on the device history. For the trap energies values between 0.6 and $0.9 \mathrm{eV}$ for peak 3 and between 0.9 and $1.0 \mathrm{eV}$ for peak 4 have been obtained. These energies are similar to the activation energy obtained from temperature dependent conductivity and mobility measurements. ${ }^{16,17}$ The small width of the TSC peaks 3 and 


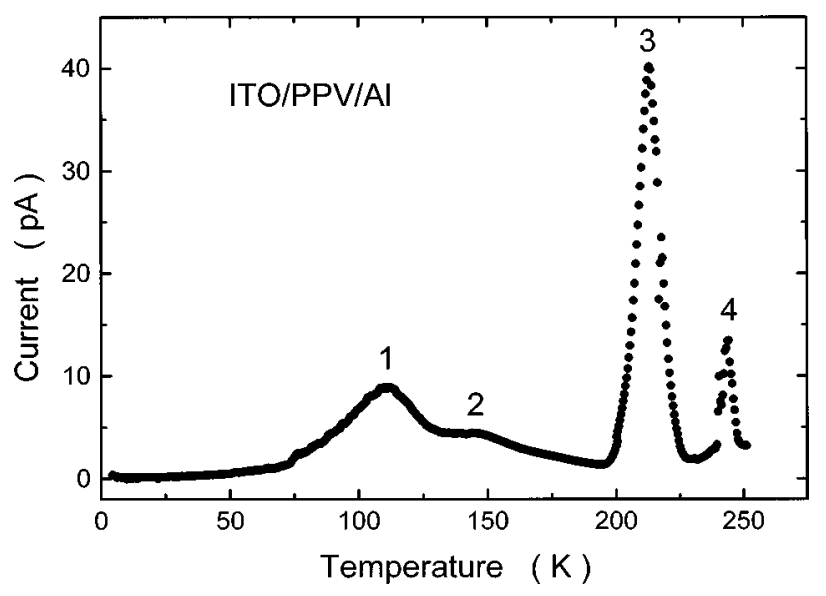

FIG. 5. Complete TSC spectrum of an ITO/PPV/Al device (PPV converted in $\mathrm{Ar}$ atmosphere).

4 in Fig. 5 indicates the existence of rather distinct trap levels. Nevertheless, a distribution of trap states cannot be fully excluded. However, the data analysis used here does not allow to make a quantitative estimate of the width of the distribution.

Motivated by the fact that the electrical conductivity of PPV is very sensitive to oxygen exposure (as discussed in the introduction), we have studied the influence of air on the deep trap states. In Fig. 6 a series of TSC spectra of a Au/ PPV/Al sample are shown as the device was exposed to different environmental conditions. After preparation (in air) the thermally stimulated current showed at least two distinct peaks at around 230 and $240 \mathrm{~K}$. After the device had been kept at ambient atmosphere for one day, one broad peak structure with an increase of current by about a factor of 6 was measured. The individual peaks cannot be resolved, however, weak shoulders indicate still a superposition of several TSC peaks. A quantitative analysis of these TSC peaks is difficult and not very accurate because of the superposition of several peaks and dynamical changes during the experiment. Approximations give values for the trap depths of these environmentally induced traps in the same range as

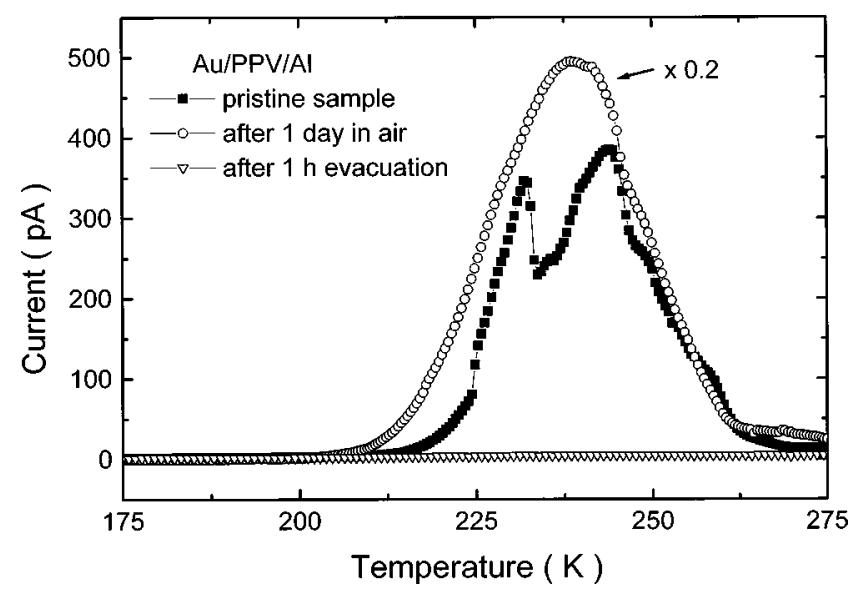

FIG. 6. TSC spectra of a Au/PPV/Al device obtained as prepared, after one day storage in air and after $1 \mathrm{~h}$ evacuation. Data of the sample stored in air (O) are divided by a factor of 5 for better visualization. those in Fig. 5. The density of releasable traps has increased while the device was stored in air up to $4 \times 10^{17} \mathrm{~cm}^{-3}$. This density is roughly an order of magnitude higher than the density obtained for the ITO induced peaks discussed before. After this experiment, the sample was exposed to vacuum $\left(10^{-4}\right.$ mbar $)$ for $1 \mathrm{~h}$. In the succeedingly measured TSC spectra, the peaks had disappeared below the measurement limit. By exposing the sample to pure oxygen the formation of the peak structure could be observed again. This experiment illustrates the reversibility of the reaction with oxygen. Thus the major amount of traps caused by oxidation are due to loosely bound agents instead of covalent bonds. The large trap density of the order of $10^{17} \mathrm{~cm}^{-3}$ explains the conductivity increase when pristine PPV is exposed to air. ${ }^{3}$

\section{Comparison with other TSC measurements on PPV}

Earlier TSC measurements on PPV have been reported by Onoda et al. ${ }^{22}$ who investigated the influence of film stretching on trap states. Nguyen et al..$^{23}$ used this method to study the conduction mechanism in PPV films and interfacial effects at PPV-metal contacts. Both groups performed their investigations on device structures which were different to the ones investigated in this publication. Onoda et al. found two TSC peaks in free standing films corresponding to trap energies of about 0.36 and $0.55 \mathrm{eV}$. The origin of the peaks is explained by impurities caused by the precursor route and by structural disorder of the PPV. Nguyen et al. fabricated metal/PPV/metal decives to measure TSC spectra. They detected up to three peaks with energies of about $0.36,0.68$ and $0.82 \mathrm{eV}$ which they attributed to interface states at the PPV/ metal electrode and a bulk trap level which is responsible for the charge transport in PPV.

A direct comparison with our results is difficult since it is known that the electrical properties of PPV films and devices depend on the details of the precursor synthesis and the conversion conditions. The measured trap energies are comparable to the deep traps determined in this work although the microscopic origin seems to be different. The absence of shallow traps in the literature is understandable because ITO was not used as substrate material.

\section{SUMMARY}

Our results show that the method of thermally stimulated currents can be usefully applied to LEDs based on PPV. In samples prepared from precursor PPV two kinds of traps could be detected. Trapping sites with deep levels between 0.6 and $1 \mathrm{eV}$ and densities up to $10^{17} \mathrm{~cm}^{-3}$ could be attributed to the oxidizing effect of air, i.e., most likely oxygen. The second group of traps consisting of two trap states with an average trap depth of about $0.03-0.06$ and $0.13-0.18 \mathrm{eV}$ is caused by a chemical reaction of the ITO substrate with $\mathrm{HCl}$ developing during the thermal elimination reaction of the PPV precursor. The estimated trap density is comparable to the value measured by the capacitance-voltage analysis. Carriers in these states are easy to ionize which leads to a depletion layer at the polymer-metal $(\mathrm{Ca}, \mathrm{Al})$ interface similar to a Schottky diode. In contrast, Au/PPV devices show no peak structures in this energy range indicating that there is 
no doping by the substrate and therefore no significant capacitance-voltage dependence. As a consequence Au/PPV devices do not exhibit Schottky-like behavior. The TSC measurements thus give a valuable contribution to the understanding of charge transport and the device physics in PPV light-emitting devices.

\section{ACKNOWLEDGMENTS}

The authors thank J. Gmeiner for polymer synthesis and M. Herold and W. Rieß for valuable discussions. Financial support from the Fonds der Chemischen Industrie and from the Bayerische Forschungsstiftung (FOROPTO) is gratefully acknowledged.

${ }^{1}$ I. Murase, T. Ohnishi, T. Noguchi, and M. Hirooka, Polym. Commun. 25, 327 (1984).

${ }^{2}$ D. Gagnon, J. D. Capistran, F. E. Karasz, R. W. Lenz, and S. Antoun, Polymer 28, 567 (1987)

${ }^{3}$ S. Tokito, T. Tsutsui, and S. Saito, Polym. Commun. 27, 333 (1986).

${ }^{4}$ J. Gmeiner, S. S. Karg, M. Meier, W. Rieß, P. Strohriegl, and M. Schwoerer, Acta Polym. 4, 201 (1993).

${ }^{5}$ M. Herold, J. Gmeiner, C. Drummer, and M. Schwoerer, J. Mater. Sci. 32, 5709 (1997).
${ }^{6}$ S. Karg, M. Meier, and W. Rieß, J. Appl. Phys. 82, 1951 (1997).

${ }^{7}$ M. Meier, S. Karg, W. Rieß, J. Appl. Phys. 82, 1961 (1997).

${ }^{8}$ W. Brütting, M. Meier, M. Herold, S. Karg, and M. Schwoerer, Chem. Phys. 227, 243 (1998)

${ }^{9}$ G. Sauer, M. Kilo, M. Hund, A. Wokaun, S. Karg, M. Meier, W. Rieß, M. Schwoerer, H. Suzuki, J. Simmerer, H. Meyer, and D. Haarer, Fresnius J. Anal. Chem. 353, 642 (1995).

${ }^{10}$ M. Herold, J. Gmeiner, and M. Schwoerer, Acta Polym. 45, 392 (1994).

${ }^{11}$ J. Slowik, J. Appl. Phys. 47, 2982 (1976).

${ }^{12}$ R. Eiermann, W. Hofberger, and H. Bässler, J. Non-Cryst. Solids 28, 415 (1978)

${ }^{13}$ I. Glowacki and J. Ulanski, J. Appl. Phys. 78, 1995 (1995).

${ }^{14}$ E. Fabre and R. N. Bhargava, Appl. Phys. Lett. 24, 322 (1974).

${ }^{15}$ I. Chen, J. Appl. Phys. 47, 2988 (1976).

${ }^{16}$ S. Paasch, W. Rieß, S. Karg, M. Meier, and M. Schwoerer, Synth. Met. 67, 177 (1994).

${ }^{17}$ E. Lebedev, T. Dittrich, V. Petrova-Koch, S. Karg, and W. Brütting, Appl. Phys. Lett. 71, 2686 (1997).

${ }^{18}$ R. Haering and E. N. Adams, Phys. Rev. 117, 451 (1960).

${ }^{19}$ T. Cowell and J. Woods, Br. J. Appl. Phys. 18, 1045 (1967).

${ }^{20}$ S. Sze, Physics of Semiconductor Devices (Wiley, New York, 1981).

${ }^{21}$ J. Scherbel, P.H. Nguyen, G. Paasch, W. Brütting, and M. Schwoerer, J. Appl. Phys. 83, 5045 (1998).

${ }^{22}$ M. Onoda, D. H. Park, and K. Yoshino, J. Phys.: Condens. Matter 1, 113 (1989).

${ }^{23}$ T. P. Nguyen, V. H. Tran, and V. Massardier, J. Phys.: Condens. Matter 5, 6243 (1993) 